\title{
Editorial: Quasars at All Cosmic Epochs
}

\author{
Paola Marziani ${ }^{1 *}$, Ascensión Del Olmo ${ }^{2}$, Mauro D'Onofrio ${ }^{3}$ and Deborah Dultzin ${ }^{4}$ \\ ${ }^{1}$ National Institute for Astrophysics (INAF), Padua Astronomical Observatory, Padua, Italy, ${ }^{2}$ Instituto de Astrofísica de \\ Andalucía (CSIC), Granada, Spain, ${ }^{3}$ Dipartimento di Fisica \& Astronomia "Galileo Galilei," Università di Padova, Padua, Italy, \\ ${ }^{4}$ Instituto de Astronomía, UNAM, Mexico City, Mexico
}

Keywords: quasars, cosmology, massive and supermassive black holes, galaxies, galaxy evolution, active galactic nuclei, accretion processes

\section{Editorial on the Research Topic}

\section{Quasars at All Cosmic Epochs}

Accretion onto massive black holes-and its associated manifestation as nuclear activity in galaxies-is among the most remarkable phenomena occurring in the Universe, and likely a key factor in galaxy evolution over a broad range of cosmic epochs. From a time when it was believed that accreting black holes were isolated sources affecting their host only within a limited sphere of influence, we have just begun to appreciate the rich and complex phenomenology induced by active nuclei in their host galaxies due to their radiative and mechanical output. Nuclear activity also presents a unique opportunity to probe spatial scales that we cannot yet resolve, as accretion related phenomena provide an immensely rich phenomenology extending from the longest radio wavelengths to the hardest $\gamma$-rays within a few parsec from the central black hole. A world of accreting black holes completely hidden at present will emerge in the next few years from the development of gravitational wave observatories. In late 2016, the time was ripe for a critical assessment of our understanding of quasars as accreting black holes and of their evolution across the cosmic time, with an eye on future developments.

This Research Topic is based on contributions presented at a meeting held in Padova in April 2017. Close to 170 participants convened in Padova from research institutes and Universities around the world. The Padova meeting was one of the largest conferences on active galactic nuclei in recent years. With 159 contributions ( 88 talks), gender balance was achieved (within 2 $\sigma$ confidence-level stochastic fluctuations expected from binomial statistics), as the percentage of women participant was $43 \%$, the percentage of women first author in a contribution $48 \%$, and the percentange of women speakers was $55 \%$.

The meeting highlighted progress-both observational and theoretical-achieved over the last decade. At the same time, many shortcomings, wishful thinkings, and never critically-reanalyzed prejudices emerged in discussions among the participants about our view of quasars and nuclear activity in general.

The organization of the e-book produced from this Research Topic reflects the temporal sequence of the meeting sessions. Each day was organized around a broad theme, focused on one or more overarching questions. On the first day, we aimed at a comprehensive overview of the main observational aspects of active galactic nuclei (AGN), with a focus on several key questions (Chapter 1). Contributions from this first day, organized into Chapter 1, discuss the most relevant photometric, spectroscopic, polarimetric, and variability observations over the full spectral energy distribution (SED), together with their interpretation, with attention to both statistical results and case studies. Overarching issues are some still-enigmatic aspects of the SED, systematic organization, and contextualization of observational properties, radio properties of 
jetted and non-jetted quasars, as well as selection effects that still affect major surveys. Chapter 2 contains contributions on accretion disk structure and wind and jet launching processes. The overarching theoretical issues (which remain open to-date) are the connections between disk structure, relativistic ejections, and continuum and line emitting regions. A satisfactory model of the broad line emitting region of quasars - to which almost 2,000 papers were dedicated over 40 years and to which a large part of the Conference was devoted-could pave the road to the use of quasars as distance indicators but remains as yet incomplete. The contributions in Chapter 3 attempt to connect theory and observation. The overarching questions remain, why do the radio properties of quasars divide the quasar population into the two categories radio quiet and radio loud? What is the connection between disk structure, relativistic ejections, and emitting regions structure? We think that one of the more remarkable outcomes of the conference was the realization of the many observational manifestations of accretion in quasars in a broad range of luminosity and cosmic epochs, and of their dependence on accretion parameters such as mass, dimensionless accretion rate, and spin. As the accreting black hole systems only possess axial symmetry and not spherical symmetry, many observational properties are affected by the viewing angle. Our ability to connect observational to physical and aspect parameters is still lacunose but, if filled, the importance could be comparable to the development of the Hertzspung-Russell diagrams for stars. We miss an equivalent for quasars, although prospective improvements are in sight. Significant attention was devoted to a promising approach, developed by Jack Sulentic, his collaborators, and other research groups, based on the analysis of the "first eigenvector" of a 4D correlation space of quasar properties.

Chapter 4 and in part Chapter 5 are devoted to the role of nuclear activity in shaping the evolution of galaxies. Feedback and environment of active nuclei are seen in the suggested black hole and host galaxy coevolution, but open issues still involve the interplay of black hole fueling (strongly influenced by the environment over cosmic ages), star formation and feedback. The overarching question posed at the meeting was: which are the strongest evidences and the state-of-the-art modeling, and which are the tests and surveys, both observational and theoretical, that can lead to progresses in our understanding of fueling and feedback processes on all scales?

Chapter 5 discussed some interesting developments having taken place in the last two years, as we seek to learn from the observations of the increasing number of high redshift quasars. An open issue remains the role of quasars at the epoch of re-ionization. A more basic question is the role of first seed quasars and their relation to galaxy formation. The overarching question remains whether we understand quasar evolution beyond selection effects.

The last contributions deal with the possibility to exploit quasars as helpful distance indicators, a possibility that was found closely linked to an improvement in our understanding of the quasar emitting region structure and dynamics. Major issues and the recent developments, including several research lines aimed at answering some of the overarching questions, are critically evaluated in the conference closing remarks by Hagai Netzer (Chapter 6).

\section{DATA AVAILABILITY STATEMENT}

Slides of most of the presentations are available at zenodo.org on the Quasars at all cosmic epochs page.

\section{AUTHOR CONTRIBUTIONS}

PM wrote the paper. The other authors gave significant contributions.

\section{FUNDING}

The conference was sponsored jointly by the National Institute for Astrophysics, the Padova Astronomical Observatory, the Department of Physics and Astronomy of the University of Padova, and the Instituto de Astrofísica de Andalucía (IAA) of the Consejo Superiór de Investigación Cientifica (CSIC) whose support is gratefully acknowldeged. We are very grateful for the facilitation and sponsorship (including the best poster contest) that was provided by the Journal Frontiers in Astronomy and Space Sciences. Sponsors Officina stellare and Springer Verlag provided conference material free-of-charge. The meeting has been part of the events meant to celebrate the 250th anniversary of the foundation of the Padova Observatory.

\section{ACKNOWLEDGMENTS}

The Editors wish to thank all participants, all members of the Scientific and Local Organizing Committee, and all speakers (see the conference website at https:/indico.ict.inaf.it/event/ $338 /$ and the conference poster). A special thank goes to the authors who submitted their contributions to the Journal and for the Conference proceedings and to the reviewers of the submitted papers for their time, their careful work, and incredible patience.

We gratefully acknowledge the kindness and professionalism of the people with the Editorial Office of Frontiers who masterfully handled several issues during the reviews of the Research Topic contributions, and especially Daisy Hessenberger and Claudio Bogazzi. We thank the Chief Editor of Frontiers in Astronomy and Space Science, Sam Finn, for a careful reading of the Editorial.

Conflict of Interest Statement: The authors declare that the research was conducted in the absence of any commercial or financial relationships that could be construed as a potential conflict of interest.

Copyright $\odot 2018$ Marziani, Del Olmo, D’Onofrio and Dultzin. This is an openaccess article distributed under the terms of the Creative Commons Attribution License (CC BY). The use, distribution or reproduction in other forums is permitted, provided the original author(s) and the copyright owner(s) are credited and that the original publication in this journal is cited, in accordance with accepted academic practice. No use, distribution or reproduction is permitted which does not comply with these terms. 